\title{
Influence of the matrix and polymerization methods on the synthesis of BC/PANi nanocomposites: an IGC study
}

\author{
Emanuel Alonso $\cdot$ Marisa Faria $\cdot$ Artur Ferreira $\cdot$ Nereida Cordeiro $\mathbb{D}$
}

Received: 15 November 2017 / Accepted: 1 March 2018/Published online: 8 March 2018

(C) Springer Science+Business Media B.V., part of Springer Nature 2018

\begin{abstract}
Inverse gas chromatography (IGC) is a technique for evaluating surface properties. The current work emphasizes the use of IGC to evaluate the surface physicochemical changes during different bacterial cellulose (BC) processing methods as well as upon polyaniline (PANi) incorporation. The processing methods (oven-drying, freeze-drying, and regeneration) caused changes in the $\mathrm{BC}$ surface group distribution, where upon freeze-drying and regeneration, a more acidic behavior is obtained, compared to oven-drying $\left(K_{\mathrm{b}} / K_{\mathrm{a}}\right.$ decreased up to $\left.24 \%\right)$. Through freeze-drying, the structural pore preservation increases $(54 \%)$ the $\mathrm{BC}$ porosity, whereas through regeneration, the porosity decreases $(23 \%)$, compared
\end{abstract}

Electronic supplementary material The online version of this article (https://doi.org/10.1007/s10570-018-1736-0) contains supplementary material, which is available to authorized users.

E. Alonso · M. Faria $\cdot$ N. Cordeiro $(\bowtie)$

LB3 - Faculty of Science and Engineering, University of Madeira, 9000-390 Funchal, Portugal

e-mail: ncordeiro@staff.uma.pt

A. Ferreira

CICECO - Aveiro Institute of Materials, Águeda School of Technology and Management, University of Aveiro, 3810-193 Aveiro, Portugal

\section{N. Cordeiro}

CIIMAR - Interdisciplinary Centre of Marine and Environmental Research, University of Porto,

4450-208 Matosinhos, Portugal to BC oven-drying. Regarding the nanocomposites, with PANi incorporation, the overall properties evaluated by IGC were significantly changed. The $\gamma_{\mathrm{s}}^{\text {total }}$ increases up to $150 \%$, indicating a more reactive surface in the nanocomposites. Also, is observed a sevenfold increase in the $K_{\mathrm{b}} / K_{\mathrm{a}}$ and a less porous surface (up to $85 \%$ ). Hence, the current work highlights the use of IGC as a viable technique to evaluate the physicochemical changes upon different BC modifications.

Keywords Bacterial cellulose - Polyaniline · Nanocomposites · Inverse gas chromatography

\section{Introduction}

The synthesis of new nanocomposites, leading to the exploration of new behaviors and functionalities, is of great importance. An array of advancements into functionalizing bacterial cellulose (BC) is presented in the literature, with the intent to create new $\mathrm{BC}$ nanocomposites (Kargarzadeh et al. 2017; Missoum et al. 2013; Moon et al. 2011). These new materials need to be extensively characterized in order make them suitable for the new application as new materials.

Bacterial cellulose/polyaniline (BC/PANi) nanocomposite is a special type of $\mathrm{BC}$-reinforced nanocomposite, with the ability to conduct electricity, 
and has been receiving increasing attention from the scientific community for the development as a novel electroresponsive material. With the incorporation of PANi into the BC network, it is expected several changes to the starting material due to the changes in the established intermolecular forces. Making use of a set of conventional analysis techniques, Alonso et al. (2017) observed that the BC membrane and polymerization method affected the final properties of the new BC-reinforced material. However, the same authors observed that the use of the inverse gas chromatography (IGC) allowed to infer similar conclusions.

IGC technique consists in the injection of probe molecules (specific molecules with known properties), under controlled experimental conditions, in order to obtain certain properties of the material (Thielmann 2004). This technique offers some advantages, such as its sensitivity and reproducibility, as well it requires low amount of material and can be run at a wide range of temperatures and humidities (Conder 2000; Thielmann 2004). IGC can provide an array of information regarding the properties and morphology of materials. In the current work, the changes occurred onto $\mathrm{BC}$ with the different BC processing methods, and PANi incorporation will be evaluated by IGC at a chemical level through the surface energy, energetic profile, and acid-base surface character, and at a morphological level through the BET surface area, diffusion analysis, and morphology indexes from the nonlinear alkanes. This way, the current work aims to use IGC as a viable alternative to other techniques used to surface characterization, regarding the physicochemical changes that occurred onto $\mathrm{BC}$ during the $\mathrm{BC}$ processing methods (oven-drying, freeze-drying, and regeneration) as well as through PANi incorporation using different polymerization methods (in situ and ex situ). The results obtained by IGC will be compared and correlated with ATR-FTIR, EDX, AFM, and SEM.

\section{Materials and methods}

$\mathrm{BC}$ production

Gluconacetobacter sp. was statically cultivated in Hestrin and Schramm (HS) medium (previously autoclaved for $15 \mathrm{~min}$ at $121{ }^{\circ} \mathrm{C}$ ) to meet the bacteria cellular requirements for cellulose production. After being incubated for 7 days at $30^{\circ} \mathrm{C}$, the membrane was removed and washed with $\mathrm{NaOH} 0.5 \mathrm{M}$, at $80{ }^{\circ} \mathrm{C}$ for $2 \mathrm{~h}$, and then neutralized with distilled water. Some BC membranes were stored at $4{ }^{\circ} \mathrm{C}$ until further use.

Preparation of the $\mathrm{BC}$ membranes

Different BC matrixes were used for the synthesis of the nanocomposites, which comprised in the use of drained, freeze-dried, and regenerated BC. Oven-dried $\mathrm{BC}$ (OD-BC) was obtained by placing a wet BC membrane on the oven at $40{ }^{\circ} \mathrm{C}$. Drained BC (D-BC) was obtained through manual pressing, removing up to $90 \%$ of water, while freeze-dried BC (FD-BC) was obtained by freezing followed by freeze-drying.

To obtain a regenerated $\mathrm{BC}$ (R-BC), a wet membrane was dried on the oven at $40{ }^{\circ} \mathrm{C}$ and cut into small pieces, and a certain amount was added to a solution of $\mathrm{LiCl} 8 \%(\mathrm{w} / \mathrm{v})$ in dimethylacetamide, obtaining a BC concentration of $0.5 \%(\mathrm{w} / \mathrm{v})$. Then, the mixture was placed in an oil bath at $110{ }^{\circ} \mathrm{C}$ for $1 \mathrm{~h}$ followed by an ultrasonic bath for $1 \mathrm{~h}$ at room temperature. The mixture was left under moderate stirring overnight, obtaining a clear viscous solution.

The regeneration process was employed by adding water in the dissolved BC solution, leaving under slow stirring $(<100 \mathrm{rpm})$ for $1 \mathrm{~h}$ to gain some firmness. Afterward, the samples were washed through dialysis for $72 \mathrm{~h}$ using a dialysis tubing (benzoylated) with a molecular weight cutoff of $2000 \mathrm{Da}$ (Sigma-Aldrich) and then oven-dried at $40{ }^{\circ} \mathrm{C}$.

\section{PANi synthesis conditions}

All polymerization reactions occurred under $24 \mathrm{~h}$ and under low stirring $(<100 \mathrm{rpm})$. The membranes were washed thoroughly until no aggregates could be observed. Then, the resulting blends were oven-dried at $40{ }^{\circ} \mathrm{C}$. A BC/ANi mass ratio of 0.10 , as well as a Ani/HCl/APS molar ratio of 1:1.2:1, was employed, following the optimal conditions reported by Wang et al. (2012). The reactions occurred under an inert atmosphere $\left(\mathrm{N}_{2}\right)$, and both monomer and persulfate aqueous solutions were dissolved in the presence of $\mathrm{HCl}$ (Riedel-de Haen). 
Preparation of the $\mathrm{BC} / \mathrm{PANi}$ membranes

Different methods were used to obtain different $\mathrm{BC} /$ PANi membranes, using different $\mathrm{BC}$ matrixes (drained, freeze-dried, and regenerated) as well as the different polymerization methods (in situ and ex situ).

The first set of BC/PANi nanocomposites was obtained by using a $\mathrm{BC}$ membrane [either drained (D) or freeze-dried (FD)], and letting stand for $1 \mathrm{~h}$ with an aniline solution in order to incorporate the monomer prior to the addition of the oxidizing agent [ammonium persulfate (APS, Sigma-Aldrich)]. This way, it is expected that the polymerization of aniline occurs inside of the membranes, being considered an in situ polymerization. This way, the BC/PANi nanocomposites obtained were named D-IS and FDIS, being the prefix the BC used (drained or freezedried) and the suffix (IS) the type of polymerization used (in situ).

Likewise, the second set of BC/PANi nanocomposites obtained comprised in the use of a $\mathrm{BC}$ membrane (drained or freeze-dried), but this time aniline and APS were added at the same time. This way it is expected that the polymerization reaction would occur outside of the membrane and thus, being considered ex situ polymerization. The nomenclature of the second set of BC/PANi nanocomposites followed the same pattern as the first set, being named accordingly D-ES and FD-ES, where the prefix refers the type of BC used (drained or freeze-dried) and the suffix (ES) to the type of polymerization used (ex situ).

Then, the third set consists in the in situ polymerization of aniline in the presence of dissolved BC. Pure aniline was added into dissolved BC (see "Preparation of the BC membranes" section), letting under low stirring $(<100 \mathrm{rpm})$ for $4 \mathrm{~h}$ in order for full homogenization. Then, it was added of the oxidizing agent (APS) aqueous solution, which not only made aniline polymerize but also made $\mathrm{BC}$ to regenerate. This way, a 2-in-1 step method was employed, regenerating BC at the same time of PANi synthesis. Following the same nomenclature as the other samples, this membrane was called R-IS, being the prefix (R) a reference to the $\mathrm{BC}$ present (regenerated $\mathrm{BC}$ ) and the suffix (IS) a reference to the polymerization method employed (in situ).
Inverse gas chromatography (IGC)

IGC measurements were carried out on a commercial inverse gas chromatograph (Surface Measure Systems London, UK), equipped with both FID and TCD detector. The system is automatized with the Software SMS IGC Controller v1.8. Silanized glass columns with $3 \mathrm{~mm}$ inner diameter and $300 \mathrm{~mm}$ length were used (dimethyldichlorosilane, Repelcote BDH, UK), packing the sample through vertical tapping. The samples were conditioned overnight followed by $2 \mathrm{~h}$ at measurement temperature to stabilize, using a helium flow.

Dispersive surface energy measurements were carried using a series of $n$-alkanes, from octane to undecane. For specific surface energy measurements, tetrahydrofuran, dichloromethane, ethyl acetate, acetonitrile, and ethanol were used. In all current analyses, a concentration of $0.2 \mathrm{p} / \mathrm{p}_{0}$ was employed as well carried with a flow rate of $10 \mathrm{~mL} / \mathrm{min}$ at $25^{\circ} \mathrm{C}$ for the BC matrixes, $40-55{ }^{\circ} \mathrm{C}$ for the nanocomposites and at $70-85{ }^{\circ} \mathrm{C}$ for PANi sample. The higher temperatures applied for the nanocomposites and PANi powder are due to the strong interactions of the probes with the samples.

The probes were supplied by Sigma-Aldrich, with analytic grade (>99\%). Methane was used as an inert reference gas, and helium was used as the carrier gas, both supplied by Air Liquide Company, with purity above $99 \%$. The physical constants used, and the theoretical background, can be found in supplementary material.

Scanning electronic microscopy coupled with energy-dispersive X-ray spectroscopy (SEMEDX)

The samples were mounted and gold-coated in preparation for SEM-EDX imaging analysis, performed using a scanning electron microscope SU3500. SEM images were obtained using a magnification of $3000 \times$. The EDX analysis was performed under an accelerated voltage of $5 \mathrm{kV}$, with the aim to identify the chemical compositions of samples at the surface, determining the weight percentages (wt \%) of elements $\mathrm{C}, \mathrm{O}, \mathrm{N}, \mathrm{S}$ and $\mathrm{Cl}$. 
Atomic force microscopy (AFM)

To evaluate the surface topography of the samples, the AFM analysis was employed by an atomic force microscope (AFM, Solver PRO, NT-MDT, Russia) in tapping mode in air atmosphere. Samples were scanned with the standard Si (silicon) cantilever with a force constant of $22 \mathrm{~N} / \mathrm{m}$, at a resonance frequency of $325 \mathrm{kHz}$ (tip radius was $10 \mathrm{~nm}$ and the tip length was $95 \mu \mathrm{m}$ ), using a scan rate of $1.3 \mathrm{~Hz}$. The surface roughness $\left(R_{\mathrm{q}}\right)$ was measured from representative images at $5 \times 5 \mu \mathrm{m}^{2}$ area, which corresponds to the average value of the surface height.

\section{Statistical analysis}

The statistical analysis of the data was carried using the IBM SPSS Statistics 23 software. Differences in the measurements of a given parameter were assessed by one-way analysis of variance (ANOVA), followed by a Tukey's post hoc analysis. For IGC, the error of the measurements was of $3 \%$, and the average values were presented. $p$ values of $<0.05$ were considered statistically different.

The agglomerative method was also applied to classify the examined materials according to their similarity, using the IGC data. Similarities were calculated from the linkage distance (Euclidean distance) using the Ward method. The parameters used for the agglomerative method were the following: $\gamma_{\mathrm{s}}^{\mathrm{d}}$; $\gamma_{\mathrm{s}}^{\mathrm{sp}} ; \gamma_{\mathrm{s}}^{\text {total }} ; \Delta G^{\mathrm{sp}}$ of ethanol $(\mathrm{EtOH})$, tetrahydrofuran (THF), acetonitrile, dichloromethane, ethyl acetate; $\Delta G^{\mathrm{sp}}(\mathrm{EtOH} / \mathrm{THF})$ ratio, adsorption potential maximum of $n$-octane, THF and EtOH $K_{\mathrm{b}} / K_{\mathrm{a}}$ ratio, $S_{\mathrm{BET}}$, $D p$, and morphology indexes of 2,2,4-trimethylpentane and cyclooctane.

\section{Results and discussion}

Effect of BC membranes processing methods

The BC membranes had a $\gamma_{\mathrm{s}}^{\mathrm{d}}$ between 34.58 and $37.65 \mathrm{~mJ} / \mathrm{m}^{2}$ at $25^{\circ} \mathrm{C}$ (Table 1), close to the values found by Castro et al. (2015) and Ferguson et al. (2016), which reported values of 39.6 and $42.3 \mathrm{~mJ} / \mathrm{m}^{2}$, at 20 and $30^{\circ} \mathrm{C}$, respectively. No significant differences $(p<0.05)$ were found in the $\gamma_{\mathrm{s}}^{\mathrm{d}}$ values of the different $\mathrm{BC}$, which indicates that its surface reactivity with the apolar molecules was not affected by the different processing methods.

To know the influence of the BC processing method in the surface active sites distribution, the heterogeneity profile of $n$-octane was assessed (Fig. 1). It is observed that OD-BC present higher adsorption potential maximum $\left(A_{\max }\right)$, meaning the presence of active sites with higher energy; however, it presents lower number of active sites comparatively to both FD-BC and R-BC, leading to similar $\gamma_{\mathrm{s}}^{\mathrm{d}}$ values between the $\mathrm{BC}$ membranes. Thus, even though no significant differences were observed in the $\gamma_{\mathrm{s}}^{\mathrm{d}}$ values, it was observed different in the $n$-octane heterogeneity profiles for the different $\mathrm{BC}$ membranes, indicating that different processing methods influence the group distribution at the surface.

Moreover, the effect of the temperature on the surface energy was determined $\left(\Delta \gamma_{\mathrm{s}}^{\mathrm{d}} / \Delta T\right)$. The results present a negative trend, which can be explained by the thermodynamics of adsorption, and as being an exothermic phenomenon, the increasing temperature leads to a less favorable adsorption. OD-BC presents the highest temperature dependence, while FD-BC presents the lowest. As IGC evaluates the materials at a surface level, the differences between the BC membranes indicates that the different $\mathrm{BC}$ processing methods influenced the chain structural organization on the BC surface and consequently their degrees of freedom. Thus, the results indicate that the freezedrying process give rise to a $3 \mathrm{D}$ network structure where the cellulose chains are strongly linked $(\mathrm{OH}$ bonding). In the oven-drying process, the $3 \mathrm{D}$ structure collapsed, which caused an increase in the cellulose chain degrees of freedom, making the material more susceptible to temperature changes.

The injection of polar probes provided information regarding the acid-base properties of the BC surfaces under study. Regarding the specific component of the surface energy $\left(\gamma_{\mathrm{s}}^{\mathrm{sp}}\right)$, the different $\mathrm{BC}$ membranes present significant changes in the $\gamma_{\mathrm{s}}^{\mathrm{sp}}$ values $(p<0.05)$. FD-BC presents the highest $\gamma_{\mathrm{s}}^{\mathrm{sp}}$ value, followed by OD-BC and R-BC. Observing the heterogeneity profile of both ethanol and tetrahydrofuran (Fig. 2), it can be concluded that it is due to the highest number of active sites with the highest energy at the FD-BC surface. 
Table 1 Surface energy and acid-base behavior of BC/PANi nanocomposites at $25^{\circ} \mathrm{C}$

\begin{tabular}{lclclll}
\hline Sample & $\gamma_{\mathrm{s}}^{\mathrm{d}}\left(\mathrm{mJ} / \mathrm{m}^{2}\right)$ & $\Delta \gamma_{\mathrm{s}}^{\mathrm{d}} / \Delta T\left(\mathrm{~mJ} / \mathrm{m}^{2} \mathrm{~K}\right)$ & $\gamma_{\mathrm{s}}^{\mathrm{sp}}\left(\mathrm{mJ} / \mathrm{m}^{2}\right)$ & $\gamma_{\mathrm{s}}^{\text {total }}\left(\mathrm{mJ} / \mathrm{m}^{2}\right)$ & $\Delta \mathrm{G}_{\mathrm{s}}^{\mathrm{sp}}(\mathrm{EtOH} / \mathrm{THF})$ & $K_{\mathrm{b}} / K_{\mathrm{a}}$ \\
\hline OD-BC & $37.65^{\mathrm{a}}$ & $-0.27^{\mathrm{a}}$ & $33.38^{\mathrm{ab}}$ & $71.03^{\mathrm{a}}$ & $0.79^{\mathrm{a}}$ & $0.75^{\mathrm{a}}$ \\
FD-BC & $35.15^{\mathrm{a}}$ & $-0.06^{\mathrm{b}}$ & $37.64^{\mathrm{b}}$ & $72.79^{\mathrm{a}}$ & $0.73^{\mathrm{ab}}$ & $0.57^{\mathrm{a}}$ \\
R-BC & $34.58^{\mathrm{a}}$ & $-0.11^{\mathrm{c}}$ & $28.51^{\mathrm{a}}$ & $63.08^{\mathrm{a}}$ & $0.70^{\mathrm{b}}$ & $0.57^{\mathrm{a}}$ \\
PANi & $119.85^{\mathrm{b}}$ & $-0.89^{\mathrm{d}}$ & $123.8^{\mathrm{c}}$ & $243.65^{\mathrm{b}}$ & $1.20^{\mathrm{cd}}$ & $3.46^{\mathrm{b}}$ \\
D-IS & $64.40^{\mathrm{c}}$ & $-0.63^{\mathrm{e}}$ & $65.97^{\mathrm{d}}$ & $130.37^{\mathrm{c}}$ & $1.20^{\mathrm{cd}}$ & $2.55^{\mathrm{c}}$ \\
FD-IS & $67.74^{\mathrm{c}}$ & $-0.68^{\mathrm{f}}$ & $88.70^{\mathrm{e}}$ & $156.44^{\mathrm{d}}$ & $1.07^{\mathrm{ef}}$ & $1.88^{\mathrm{d}}$ \\
R-IS & $51.91^{\mathrm{d}}$ & $-0.46^{\mathrm{g}}$ & $105.90^{\mathrm{e}}$ & $157.81^{\mathrm{d}}$ & $0.99^{\mathrm{f}}$ & $1.91^{\mathrm{d}}$ \\
D-ES & $63.70^{\mathrm{c}}$ & $-0.39^{\mathrm{h}}$ & $63.92^{\mathrm{d}}$ & $127.62^{\mathrm{c}}$ & $1.27^{\mathrm{c}}$ & $2.26^{\mathrm{e}}$ \\
FD-ES & $75.00^{\mathrm{e}}$ & $-0.75^{\mathrm{i}}$ & $88.88^{\mathrm{e}}$ & $163.88^{\mathrm{d}}$ & $1.12^{\mathrm{de}}$ & $3.77^{\mathrm{f}}$ \\
\hline
\end{tabular}

Values in same column not sharing a common superscript are statistically different $(p<0.05)$

$\gamma_{\mathrm{s}}^{\mathrm{d}}$, dispersive component of surface energy; $\gamma_{\mathrm{s}}^{\mathrm{sp}}$, specific component of the surface energy; $\gamma_{\mathrm{s}}^{\text {total }}$, total surface energy; $\Delta \gamma_{\mathrm{s}}^{\text {total }} / \Delta T$, temperature dependence of the surface energy; $\Delta G_{\mathrm{s}}^{\mathrm{sp}}$ (EtOH/THF), ratio between the Gibbs free energy of ethanol and tetrahydrofuran; $K_{\mathrm{b}} / K_{\mathrm{a}}$, ratio between the basic and acidic surface constants

Fig. 1 Heterogeneity profile of $n$-octane from $\mathrm{BC}$ membranes and $\mathrm{BC} / \mathrm{PANi}$ nanocomposites at $25{ }^{\circ} \mathrm{C}$

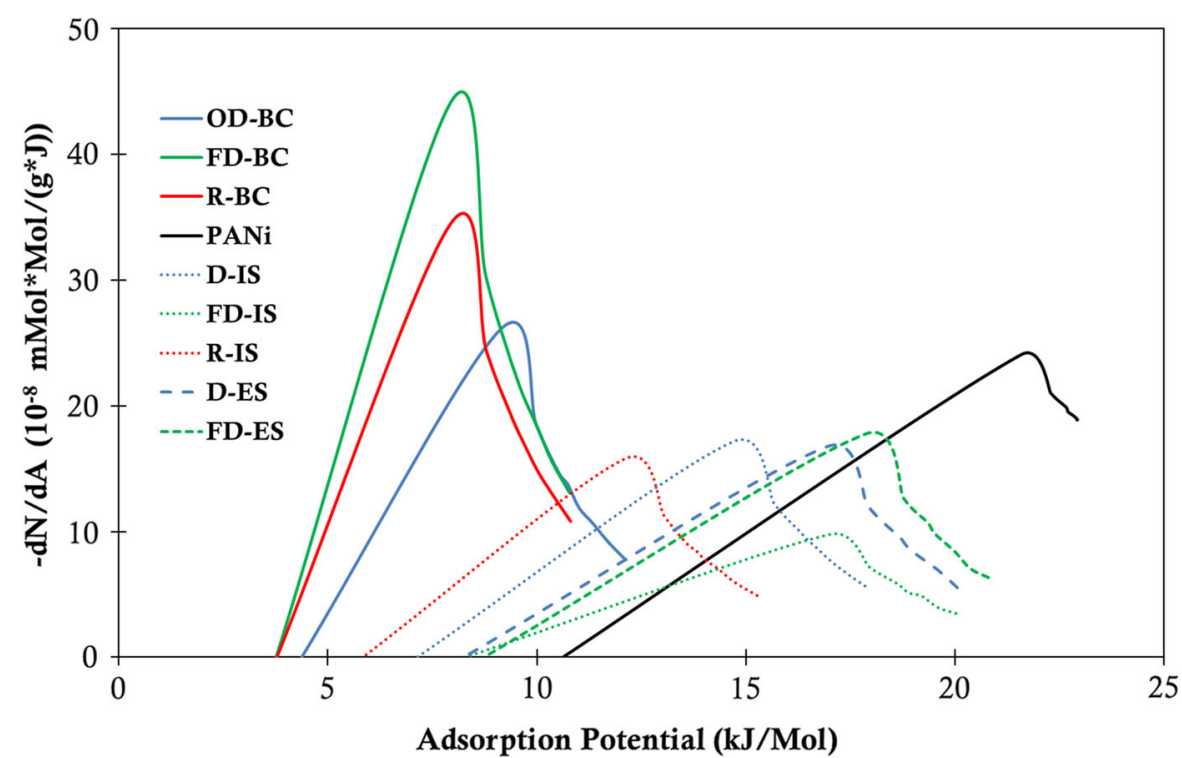

Adsorption Potential (kJ/Mol)
Thus, these results indicate that different processing methods influenced the surface group orientation, giving rise to membranes with different reactive surfaces, where FD-BC was the most reactive surface for polar probes, followed by OD-BC and R-BC.

Differences in the $\Delta G_{\mathrm{s}}^{\mathrm{sp}}$ values of the $\mathrm{BC}$ membranes in the polar probes were obtained (Fig. 3), which indicates that the drying treatments applied on $\mathrm{BC}$ influenced the polar groups at the surface. OD-BC presented higher interactions with all the studied polar probes, followed by FD-BC and R-BC. The BC membranes surface interaction with ethanol and tetrahydrofuran probes gives an insight into the basic and acid groups present on the surface, respectively. The ratio between these two probes is given in Table 1 $\left(\Delta G_{\mathrm{s}}^{\mathrm{sp}}(\mathrm{EtOH} / \mathrm{THF})\right)$. In both FD-BC and R-BC, it is observed a more evident decrease in ethanol (up to $22 \%$ ) when compared to THF (up to $12 \%$ ), indicating an increase in the acidic surface behavior.

Knowing the cellulose chemical structure, it is expected a surface amphoteric behavior, with a predominantly acidic character, due to the electron 
Fig. 2 Heterogeneity profile of ethanol (a) and tetrahydrofuran (b) from the BC/PANi nanocomposites at $25^{\circ} \mathrm{C}$

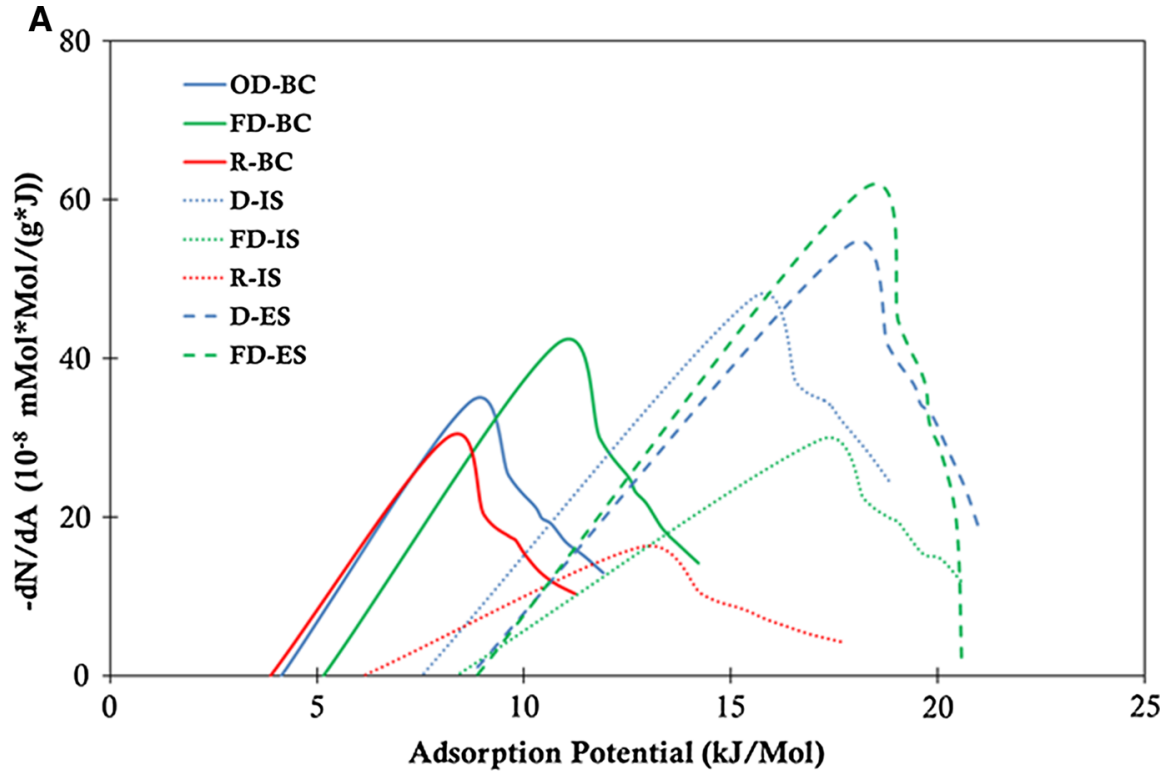

B

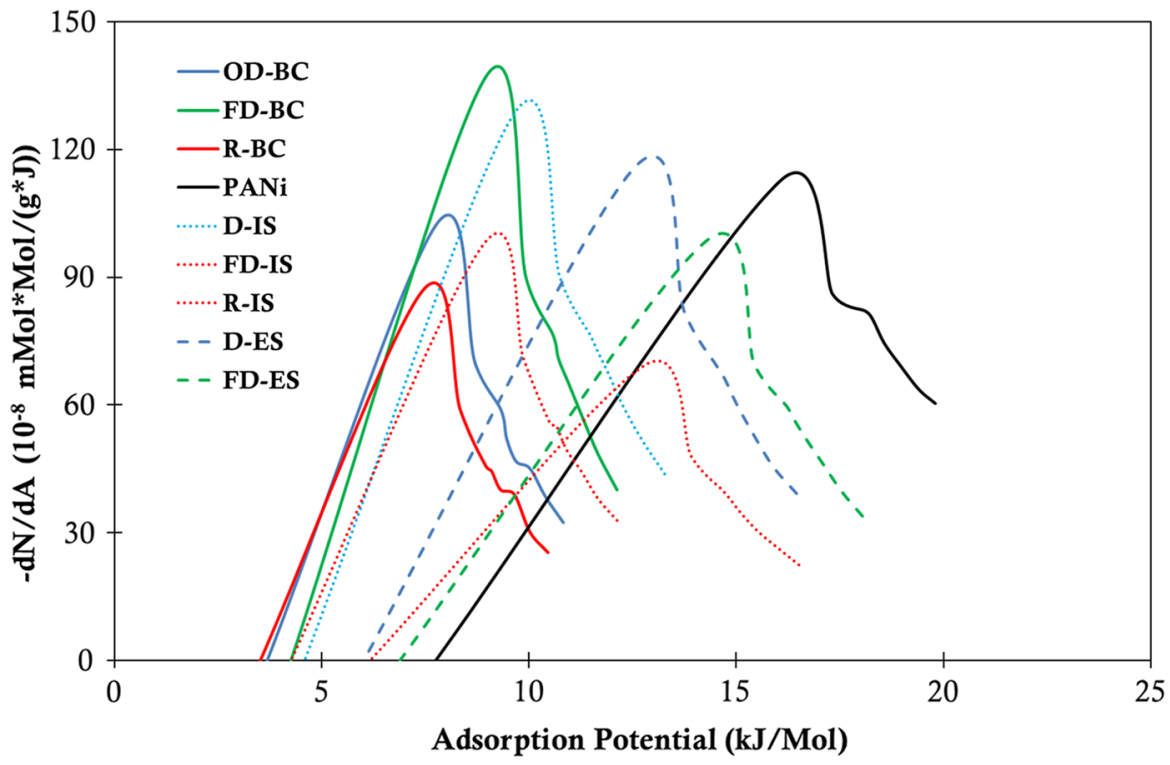

acceptor ( $\mathrm{H}$ from hydroxyl groups) and electron donor (O from glucosidic bonds and hydroxyl groups) groups. OD-BC presented a $K_{\mathrm{b}} / K_{\mathrm{a}}$ of 0.75 , which corresponds to an acidic predominant surface behavior. In both FD-BC and R-BC, the acidic behavior is more pronounced $\left(K_{\mathrm{b}} / K_{\mathrm{a}}\right.$ of 0.57$)$, meaning that a higher number of acidic groups available is found at the surface, when compared to the basic groups. This parameter corroborates the ratio between the $\Delta G_{\mathrm{s}}^{\mathrm{sp}}$ of EtOH and THF presented in Table 1. Comparatively to the literature, Castro et al. (2015) reported the same acidic behavior $\left(K_{\mathrm{b}} / K_{\mathrm{a}}\right.$ of 0.55$)$. Such phenomenon is observed due to the orientation of ether and hydroxyl groups in the surface of the BC membranes. This way, changes in the polar surface group is evidenced by the evaluation of the surface acid-base character by IGC of the different $\mathrm{BC}$ matrixes.

ATR-FTIR is usually used to know the modification on the material surface structure during a given process or treatment. In this work, the obtained ATRFTIR spectra (Alonso et al. 2017) does not evidence any modification due to the processing method. Thus, 
Fig. 3 Specific free energy of adsorption $\left(\Delta G_{\mathrm{s}}^{\mathrm{sp}}\right)$ of polar probes onto $\mathrm{BC}$ and $\mathrm{BC} / \mathrm{PANi}$ nanocomposites at $25{ }^{\circ} \mathrm{C}$

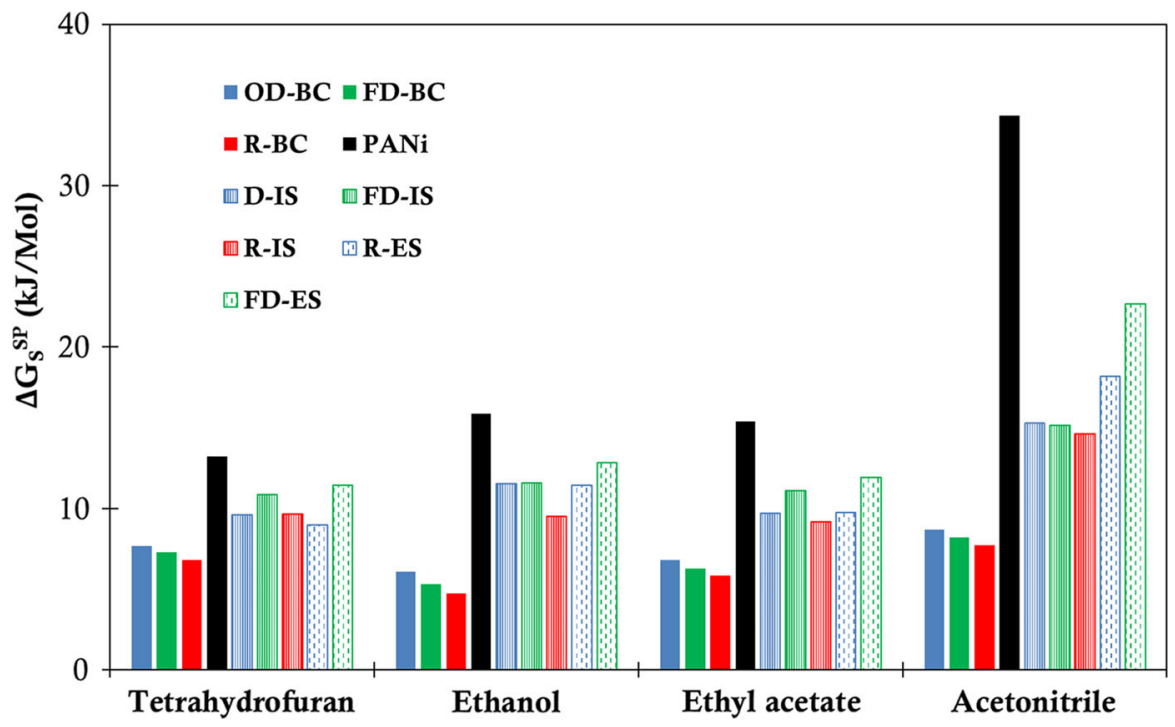

it is possible to perceive IGC high sensibility to reveal structural changes at molecular level.

The surface morphology characteristics of the BC matrixes were explored by IGC. The BC surface area $\left(S_{\mathrm{BET}}\right)$ of OD-BC, FD-BC, and R-BC were accessed through the isotherms using $n$-octane as probe (Table 2). OD-BC presents a $S_{\mathrm{BET}}$ of $4.59 \mathrm{~m}^{2} / \mathrm{g}$, which is higher than the value reported by Castro et al. (2015) of $1.94 \mathrm{~m}^{2} / \mathrm{g}$, which can be due to the different bacterial strain and biosynthesis conditions. By freeze-drying, a statistically significant increment $(p<0.05)$ of the $S_{\mathrm{BET}}$ surface area is observed due to the preservation of the porous $3 \mathrm{D}$ structure when compared to oven-drying process. This porous 3D structure makes it easier for the probes to cross the sample, increasing the $D p$ value of FD-BC. Such can be observed by the SEM micrographs (Fig. 4), where a porous structure is observed. On the other hand, the $\mathrm{BC}$ regeneration led to a smaller $S_{\mathrm{BET}}$ value, indicative of a compact structure, as shown in Fig. 4, which makes the sample less permeable to the probes, as seen by a lower $D p$.

The interaction of the probes with the surface is affected not only by the surface moiety of the sample but also by the topography of the surface (Calvet et al. 2012). The IGC morphology index gives the

Table 2 Surface area, diffusion coefficient, surface roughness, and morphology indexes from the BC matrixes and BC/PANi nanocomposites at $25^{\circ} \mathrm{C}$

\begin{tabular}{llllll}
\hline & $S_{\text {BET }}\left(\mathrm{m}^{2} / \mathrm{g}\right)$ & $D p\left(\mathrm{~cm}^{2} / \mathrm{min}\right)$ & $R_{\mathrm{q}}(\mathrm{nm})$ & \multicolumn{2}{l}{ Morphology index } \\
\cline { 3 - 5 } & & & & $2,2,4$-Trimethylpentane & Cyclooctane \\
\hline OD-BC & $4.59^{\mathrm{a}}$ & $58.10^{\mathrm{a}}$ & 25.2 & $0.12^{\mathrm{a}}$ & $1.92^{\mathrm{a}}$ \\
FD-BC & $7.05^{\mathrm{b}}$ & $76.78^{\mathrm{b}}$ & 16.2 & $0.57^{\mathrm{b}}$ & $2.24^{\mathrm{b}}$ \\
R-BC & $3.55^{\mathrm{c}}$ & $12.9^{\mathrm{c}}$ & 22.0 & $0.60^{\mathrm{b}}$ & $0.16^{\mathrm{b}}$ \\
D-IS & $1.61^{\mathrm{a}}$ & $4.04^{\mathrm{a}}$ & 40.8 & $0.60^{\mathrm{a}}$ & $0.60^{\mathrm{b}}$ \\
FD-IS & $1.07^{\mathrm{b}}$ & $0.13^{\mathrm{b}}$ & 36.5 & $0.63^{\mathrm{a}}$ & $2.00^{\mathrm{c}}$ \\
R-IS & $2.03^{\mathrm{c}}$ & $0.02^{\mathrm{b}}$ & 69.8 & $0.90^{\mathrm{b}}$ & $0.62^{\mathrm{d}}$ \\
D-ES & $1.52^{\mathrm{a}}$ & $0.64^{\mathrm{c}}$ & 39.8 & $0.43^{\mathrm{c}}$ & $0.53^{\mathrm{b}}$ \\
FD-ES & $2.04^{\mathrm{c}}$ & $0.08^{\mathrm{b}}$ & 34.8 & $0.47^{\mathrm{c}}$ & \\
\hline
\end{tabular}

Values in same column not sharing a common superscript are statistically different $(p<0.05)$

$S_{\mathrm{BET}}$, surface area; $D p$, diffusion coefficient; $R_{\mathrm{q}}$, surface roughness 

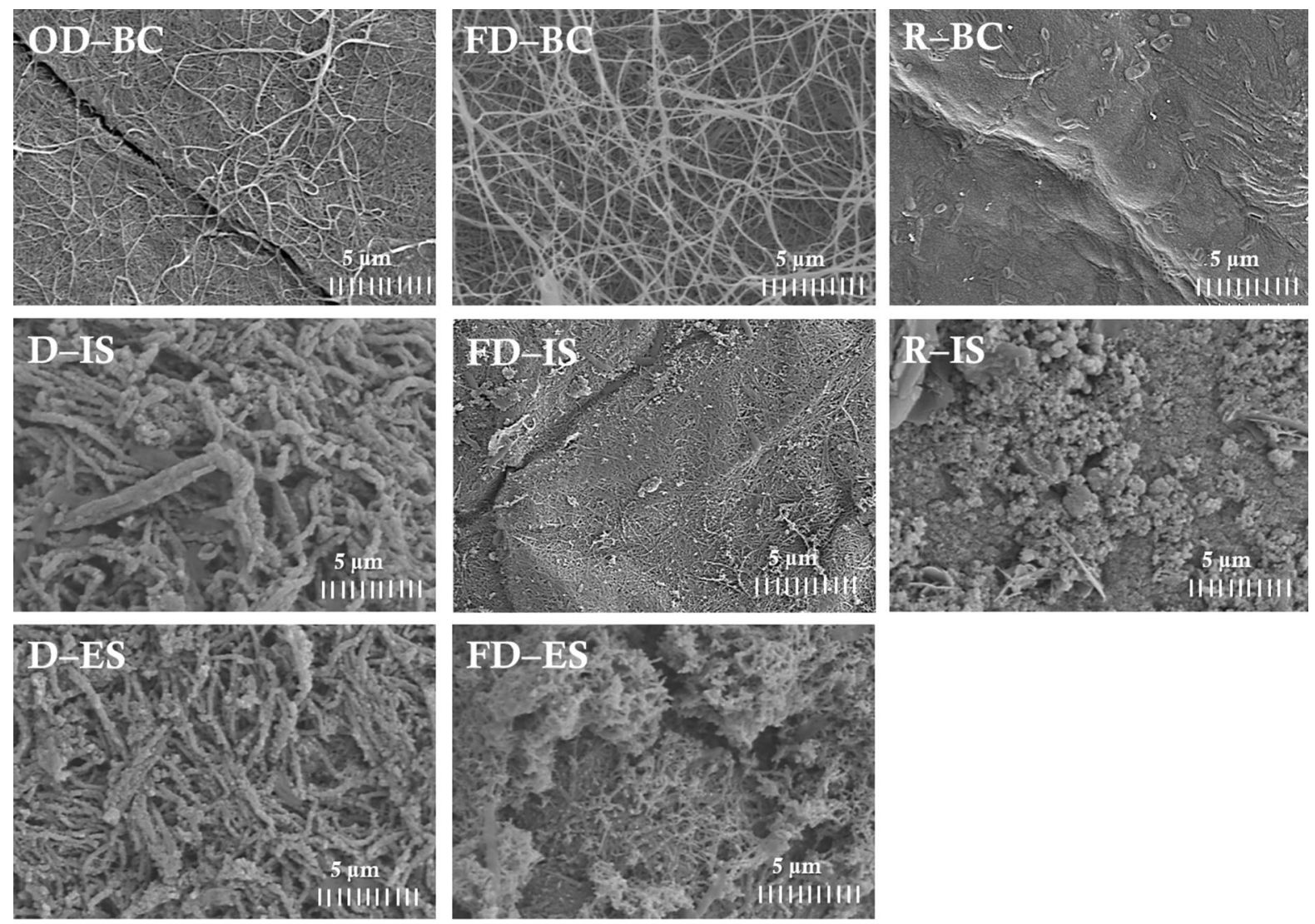

Fig. 4 SEM micrograph of the BC matrixes and BC/PANi nanocomposites

"deviation" of the sample surface from being planar, which was assessed with two probes: one branched alkane (2,2,4-trimethylpentane) and one cyclic alkane (cyclooctane), which are given in Table 2. All the BC matrixes present an adsorption phenomenon (morphology index $>1$ ) for cyclooctane, whereas for the branched alkane probe, a steric hindrance is observed (morphology index $<1$ ), which are related to the volume of the probe. Differences between the BC matrixes were observed: 2,2,4-trimethylpentane and cyclooctane presented higher morphology indexes in both FD-BC and R-BC, which indicates that the surface of OD-BC is rougher than FD-BC and R-BC. This way, the assessment of the IGC morphology indexes onto the different $\mathrm{BC}$ matrixes evidenced changes in both surface availability and morphology through the different $\mathrm{BC}$ processing methods.

The surface morphology is conventionally studied by AFM, through the determination of the surface roughness $\left(R_{\mathrm{q}}\right)$ and by SEM. The AFM images of the samples can be found in Alonso et al. (2017). The current work shows that the IGC morphology indexes agree with the $R_{\mathrm{q}}$ values obtained by AFM (Table 2), where lower $R_{\mathrm{q}}$ values are obtained in both FD-BC and R-BC (up 69\%), in comparison with OD-BC. This indicates that the fiber topography was affected by the processing methods applied. Moreover, through SEM (Fig. 4), it is observed a more porous structure on FD$\mathrm{BC}$, followed by OD-BC and R-BC, as indicated by the $S_{\mathrm{BET}}$ obtained by IGC.

In sum, through IGC results, it is evidenced that changes in the surface morphology occurred in regard to the different drying methods employed, as well through $\mathrm{BC}$ regeneration. OD-BC presented a more compact structure when compared to FD-BC which indicates a preservation of the supramolecular structure by freeze-drying. Throughout the regeneration process, the $\mathrm{BC}$ chains created a compact structure when compared to OD-BC. In terms of surface 
roughness, OD-BC presented a rougher surface than FD-BC and R-BC.

Effect of the BC membrane and polymerization methods upon PANi incorporation

Regarding the BC/PANi nanocomposites, a substantial increase in the $\gamma_{s}^{\mathrm{d}}$ values is observed upon PANi incorporation. This increase is due to the PANi presence which has high $\gamma_{\mathrm{s}}^{\mathrm{d}}$ value $\left(119.85 \mathrm{~mJ} / \mathrm{m}^{2}\right)$, compared to the different $\mathrm{BC}$ matrixes (around $36 \mathrm{~mJ} /$ $\mathrm{m}^{2}$ ). The $n$-octane heterogeneity profile of PANi (Fig. 1) presents substantially higher adsorption potential values when compared to the BC matrixes: PANi incorporation led into increased values of the active sites energy. Thus, this observed increase is a prove that PANi incorporation occurs onto the different $\mathrm{BC}$ matrixes, making the surfaces more dispersive.

To know whether PANi was present at the surface of a given material, EDX is usually applied. The results obtained from this technique (Table 3) corroborate the conclusions obtained by IGC, where it was observed a higher amount of $\mathrm{C}$ at the surface on the $\mathrm{BC} / \mathrm{PANi}$ nanocomposites in comparison with the $\mathrm{BC}$ matrixes, which contributed to the increased $\gamma_{\mathrm{s}}^{\mathrm{d}}$ in the nanocomposites.

With PANi incorporation, a statistically significant $(p<0.05)$ increase is observed on the dependence of the surface energy with the temperature $-\Delta \gamma_{\mathrm{s}}^{\mathrm{d}} / \Delta T$ (Table 1). This is due to the higher mobility of the PANi chains (more degrees of freedom). These results confirm the presence of PANi at the nanocomposites

Table 3 EDX elemental composition of the BC matrixes and BC/PANi nanocomposites

\begin{tabular}{llllll}
\hline Sample & \multicolumn{6}{l}{ Elemental composition (wt\%) } & \\
\cline { 2 - 6 } & $\mathrm{C}$ & $\mathrm{O}$ & $\mathrm{N}$ & $\mathrm{S}$ & $\mathrm{Cl}$ \\
\hline OD-BC & 52.0 & 48.0 & n.d. & n.d. & n.d. \\
FD-BC & 59.4 & 40.6 & n.d. & n.d. & n.d. \\
R-BC & 48.0 & 52.0 & n.d. & n.d. & n.d. \\
D-IS & 71.1 & 14.2 & 10.7 & 4.0 & n.d. \\
FD-IS & 70.8 & 16.9 & 10.9 & 1.4 & n.d. \\
R-IS & 69.5 & 12.9 & 8.9 & 3.5 & 5.3 \\
D-ES & 68.5 & 14.8 & 7.9 & 8.1 & 0.7 \\
FD-ES & 73.2 & 12.0 & 12.3 & 2.2 & 0.3 \\
\hline
\end{tabular}

surface, being more relevant on the FD-BC membrane. EDX results corroborate this IGC conclusion, showing the correlation between the amount of $\mathrm{N}$ and the $\Delta \gamma_{\mathrm{s}}^{\mathrm{d}} / \Delta T$ : higher $\mathrm{N}$ amount, higher PANi at the nanocomposites surface and a consequently higher temperature effect on the free chain.

Similar to $\gamma_{\mathrm{s}}^{\mathrm{d}}$, upon PANi incorporation, a significant increase in the $\gamma_{\mathrm{s}}^{\mathrm{sp}}$ values is observed. The energy of the active sites, into the heterogeneity profile of both ethanol and tetrahydrofuran, also increases (Fig. 1). However, the adsorption potential increases more significantly in ethanol (59.8-105.2\%) when compared to THF (24.2-61.5\%), resulted from the amine groups (basic character) of PANi presence at the nanocomposites surface, which increases the basic surface nature in all nanocomposites. The IGC conclusion is in accordance with the data from EDX, where upon PANi incorporation, $\mathrm{N}$ can be found at the surface (Table 3), and with ART-FTIR (Alonso et al. 2017) where PANi incorporation is visible.

The specific free energy of adsorption $\left(\Delta G_{\mathrm{s}}^{\mathrm{sp}}\right)$ of different polar probes was determined, being the data presented in Fig. 3. In general, the BC/PANi nanocomposites, presented an overall increase in the interactions with the polar probes, when compared to the $\mathrm{BC}$ matrixes, which corroborates the referred changes in the acid-base groups on the BC surface. Such happens since PANi presents higher $\Delta G_{\mathrm{s}}^{\mathrm{sp}}$ values, resulting in stronger interactions with the polar probes on the BC-reinforced materials. When comparing the different nanocomposites, higher $\Delta G_{\mathrm{s}}^{\mathrm{sp}}$ values are obtained for both FD-BC/PANi nanocomposites (IS and ES), which is an indicative of higher PANi chains at the surface in this membrane.

With PANi incorporation into $\mathrm{BC}$, the $\Delta G_{\mathrm{s}}^{\mathrm{sp}}$ of ethanol increases more significantly (up to $142 \%$ ) when compared to tetrahydrofuran (up to 57\%), suggesting a higher increase in the basic character at the surface of BC-reinforced nanocomposites.

Through the evaluation of the $K_{\mathrm{b}} / K_{\mathrm{a}}$ ratio, PANi presents the expected basic character $\left(K_{\mathrm{b}} / K_{\mathrm{a}}\right.$ of 3.46) as a result from the presence of amine groups (basic character) in the polymer chains. With PANi introduction onto the $\mathrm{BC}$ matrixes, the $\mathrm{BC}$ surface acidic behavior $\left(K_{\mathrm{b}} / K_{\mathrm{a}} 0.75\right.$ and 0.55$)$ is shifted into a basic behavior $\left(K_{\mathrm{b}} / K_{\mathrm{a}}\right.$ ranging from 1.88 to 3.77$)$, corroborating the fact that the PANi polymer can be found at the nanocomposite surfaces. Moreover, the IGC data 
are correlated with the percentage of $\mathrm{N}$ and $\mathrm{O}$ obtained by EDX (Table 3): Higher $\mathrm{N}$ and lower $\mathrm{O}$ values are associated with higher $K_{\mathrm{b}} / K_{\mathrm{a}}$ values. The percentage of $\mathrm{N}$ is associated with the amine groups of PANi, whereas the percentage of $\mathrm{O}$ is associated with the hydroxyl and ether groups of cellulose, which prove the BC coverage by PANi.

Throughout the analysis of both dispersive and specific components of the surface energy, it is observed changes in the surface reactivity of the different BC matrixes, as well as the resulting nanocomposites. This way, different $\gamma_{\mathrm{s}}^{\text {total }}$ values were obtained, and through Tukey's post hoc analysis, four groups are observed: (1) BC matrixes, (2) drained BC/ PANi nanocomposites, (3) regenerated and freezedried BC/PANi nanocomposites, and (4) PANi powder (Table 1). This indicates that different processing methods did not significantly affect the surface reactivity of $\mathrm{BC}$. On the other hand, with PANi incorporation, the $\gamma_{\mathrm{s}}^{\text {total }}$ values were significantly affected by the BC membrane employed while not influenced in the polymerization methods for the synthesis of the nanocomposites.

In terms of morphological analyses, with the incorporation of PANi, the $S_{\mathrm{BET}}$ of the different BC matrixes is drastically reduced $(64-85 \%)$. This was due to that during PANi incorporation the BC pores are obstructed, which not only decreases the area available for probes to access (decreasing the $S_{\mathrm{BET}}$ ) but also makes harder for probes to cross the sample (lower $D p$ ) (Table 2).

By statistical analyses, the $S_{\mathrm{BET}}$ has no direct correlation with the polymerization method nor with BC membrane used for the synthesis of BC/PANi nanocomposites. On the other hand, the $D p$ values on D-IS and FD-IS BC/PANi nanocomposites are higher than the values obtained in D-ES and FD-ES BC/PANi nanocomposites, respectively. R-IS BC/PANi nanocomposite presented the highest $S_{\mathrm{BET}}$ and lowest $D p$ value, which differentiates from the remaining nanocomposites, which can be due to the compact structure of R-BC whereas the high $S_{\mathrm{BET}}$ value can be due to the smaller cellulose chains (incomplete chain regeneration) and/or to the high increase in the $R_{\mathrm{q}}$ (Table 2). Thus, IGC evidences that the diffusion of the probes is influenced by the polymerization method applied, which indicates differences on the pore availability upon PANi introduction through different polymerization methods.

The IGC morphology index of the BC/PANi nanocomposites was assessed, observing on most probes a steric hindrance phenomenon (morphology index < 1) (Table 2). Through Tukey's post hoc analysis, the data obtained by 2,2,4-trimethylpentane fall into three groups: ex situ nanocomposites, in situ nanocomposites, and R-IS BC/PANi nanocomposites. This indicates that this probe did not detect changes in the pore availability using different BC matrixes, but it was influenced by the polymerization method used. For cyclooctane, when comparing the nanocomposites to the BC matrixes, a decreased surface availability of the nonlinear alkanes is observed, shifting from an adsorption (BC matrixes) to a steric hindrance (BC/ PANi nanocomposites) behavior. This observation agrees with the $S_{\mathrm{BET}}$ and $D p$ results (Table 2), since blocking the $\mathrm{BC}$ pores results in a more compact surface, leading into lower $S_{\mathrm{BET}}$ values. Also, in situ nanocomposites (D-IS and FD-IS) present higher morphology indexes in comparison to ex situ nanocomposites (D-ES and FD-ES). Thus, cyclooctane evidenced differences in the surface availability using different polymerization methods and BC matrixes.

Through AFM and SEM, it was observed that the $\mathrm{BC} / \mathrm{PANi}$ nanocomposites are rougher than the BC matrixes, which explains the steric hindrance observed for cyclooctane. R-IS depicted the highest surface roughness of all nanocomposites which interestingly presented an adsorption behavior for cyclooctane (morphology index $>1$ ). This indicates that the surface topography of the sample contributed to the adsorption of the probe, as also seen through $S_{\mathrm{BET}}$ measurement. Thus, it was evidenced differences in the surface morphology through the use of different polymerization methods (in situ and ex situ polymerization).

Cluster analysis

As observed throughout the current work, IGC evidenced changes on $\mathrm{BC}$ upon the different $\mathrm{BC}$ processing methods as well through PANi incorporation. Moreover, it has been evidenced the influence of the $\mathrm{BC}$ membrane and polymerization methods in the surface properties of the nanocomposites. Cluster analysis was performed in order to obtain meaningful 


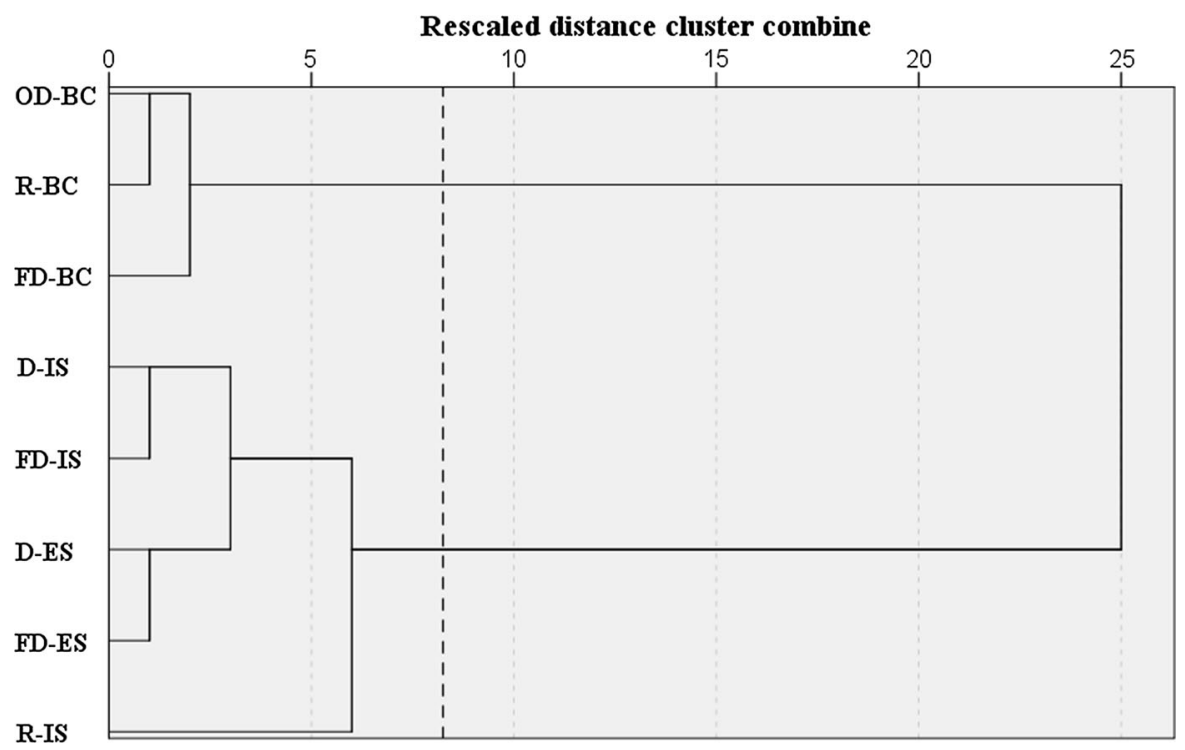

Fig. 5 Dendrogram of the BC matrixes and BC/PANi nanocomposites using the IGC parameters

visual data through the similarity between the samples, using only the IGC data (Fig. 5). The Sneath criterion (dashed line) and the agglomeration schedule were used to determine the number of relevant clusters. This way, it is observed that the samples cluster into two groups: $\mathrm{BC}$ matrixes and $\mathrm{BC} / \mathrm{PANi}$ nanocomposites. The resulting clusters represent meaningful information, indicating that through PANi incorporation the $\mathrm{BC}$ surface properties are greatly influenced.

Looking to the $\mathrm{BC}$ matrixes, both $\mathrm{R}-\mathrm{BC}$ and $\mathrm{OD}-$ $\mathrm{BC}$ are closer (therefore more similar) in comparison with FD-BC, which can be due to the similarity in the data obtained by $S_{\mathrm{BET}}, D p$, as well from the adsorption potential maxima of $n$-octane, tetrahydrofuran, and ethanol.

Interestingly, inside the cluster of the $\mathrm{BC} / \mathrm{PANi}$ nanocomposites, it is evidenced the similarity between the in situ and ex situ polymerization. This similarity is due to the data from $\gamma_{\mathrm{s}}^{\mathrm{d}}, D p$, and from the morphology indexes of cyclooctane that evidenced that the final properties of the nanocomposites are more influenced by the polymerization method, in comparison with the BC membrane employed. R-IS is highlighted as the least similar of the nanocomposites which is due to the most divergent results obtained from the following data: $\gamma_{\mathrm{s}}^{\mathrm{d}}, \Delta G_{\mathrm{s}}^{\mathrm{sp}}$ values of dichloromethane and ethanol $S_{\mathrm{BET}}, D p$, the morphology index of the probes, as well as from the adsorption potential maxima of $n$-octane, tetrahydrofuran, and ethanol.

\section{Conclusion}

The current work shows that IGC is a powerful versatile technique, used as an alternative to the recurring usual characterization techniques, to detecting changes in the $\mathrm{BC}$ properties throughout the use of different modifications processes. Changes with the $\mathrm{BC}$ processing methods were more evident in the morphology results, obtaining a more porous $\mathrm{BC}$ when freeze-drying, while obtaining a more compact material upon $\mathrm{BC}$ regeneration. By oven-drying $\mathrm{BC}$, a rougher surface is observed. PANi incorporation led to significant changes in the $\mathrm{BC}$ properties, where the highly energetic and basic properties of PANi were observed in the BC/PANi nanocomposites. Moreover, the $\mathrm{BC}$ pores were obstructed due to PANi incorporation, which are supported by the increase in the morphology indexes by the different nonlinear alkane probes. The cluster analysis demonstrated that the IGC not only was able to evidence changes upon PANi incorporation, as it also evidenced the effect of the polymerization method. The IGC proved that it can be used as a unique technique to evaluate the changes at a chemical level through the surface energy and acid/ basic character and at a morphological level through the study of the gas diffusion and surface area.

Acknowledgments The authors would like to thank the Programa Nacional de Re-equipamento Científico, POCI 2010, for sponsoring IGC work (FEDER and Foundation for 
Science and Technology). Moreover, the help of Tomásia Fernandes and Igor Fernandes (Madeira University) in the laboratory work was appreciated.

\section{References}

Alonso E, Faria M, Mohammadkazemi F, Resnik M, Ferreira A, Cordeiro N (2017) Conductive bacterial cellulosepolyaniline nanocomposites: influence of the matrix and synthesis conditions. Carbohydr Polym 183:254-262

Balard H (1997) Estimation of the surface energetic heterogeneity of a solid by inverse gas chromatography. Langmuir 13:1260-1269

Brendlé E, Papirer E (1997) A new topological index for molecular probes used in inverse gas chromatography for the surface nanorugosity evaluation. J Colloid Interface Sci 194:207-216

Calvet R, Confetto S, Balard H, Brendlé E, Donnet J (2012) Study of the interaction of polybutadiene/fillers using inverse gas chromatography. J Chromatogr A 1253:164-170

Castro C, Cordeiro N, Faria M, Zuluaga R, Putaux J, Filpponen I, Velez L, Rojas O, Gañán P (2015) In-situ glyoxalization during biosynthesis of bacterial cellulose. Carbohydr Polym 126:32-39

Conder J (2000) Physicochemical measurements: gas chromatography. In: Cooke M, Poole C (eds) Encyclopedia of separation science. Academic Press, Detroit, pp 3808-3815

Cordeiro N, Gouveia C, Moraes A, Amico S (2011) Natural fibers characterization by inverse gas chromatography. Carbohydr Polym 84:110-117

Ferguson A, Khan U, Walsh M, Lee K, Bismarch A, Shaffer M, Coleman J, Bergin S (2016) Understanding the dispersion and assembly of bacterial cellulose in organic solvents. Biomacromolecules 17:1845-1853

Fowkes F (1964) Attractive forces at interfaces. Ind Eng Chem 56:40-52

Ghazali M, Nawawi M (2000) Diffusion coefficient estimations by thin-channel column inverse gas chromatography: preliminary experiments. Pertan J Sci Technol 8:1-18

Goss K (1997) Considerations about the adsorption of organic moelcules from the gas phase to surfaces: implication for inverse gas chromatography and the prediction of adsorption coefficients. J Colloid Interface Sci 190:241-249

Gutmann V (1978) The donor-acceptor approach to molecular interactions. Springer, New York

Jackson P, Huglin M (1995) Use of inverse gas chromatography to measure diffusion coefficients in crosslinked polymers at different temperatures. Eur Polym J 31:63-65

Kargarzadeh H, Mariano M, Huang J, Lin N, Ahmad I, Alain D, Thomas S (2017) Recent developments on nanocellulose reinforced polymer nanocomposites: a review. Polymer 132:368-393

Missoum K, Belgacem M, Bras J (2013) Nanofibrillated cellulose surface modification: a review. Materials 6:1745-1766

Moon R, Martini A, Nairn J, Simonsen J, Youngblood J (2011) Cellulose nanomaterials review: structure, properties and nanocomposites. Chem Soc Rev 40:3941-3994

Mukhopadhyay P, Schreiber H (1995) Aspects of acid-base interactions and use of inverse gas chromatography. Colloids Surf A Physicochem Eng Asp 100:47-71

Oss V (1988) Interfacial Lifshitz-van der Waals and polar interactions in macroscopic system. Chem Rev 88:927-941

Riddle F, Fowkes F (1990) Spectral shifts in acid-base chemistry. 1-Van der Waals contributions to acceptor numbers. J Am Soc 112:3259-3264

Schultz J, Lavielle L, Martin C (1987) The role of the interface in carbon-fibre epoxy composites. J Adhes 23:45-60

Sen A (2005) Inverse gas chromatography. Defense Scientific Information \& Documentation Centre, New Delhi

Thielmann F (2004) Introduction into the characterization of porous materials by inverse gas chromatography. J Chromatogr A 1037:115-123

Voelkel A, Strzemiecha B, Adamska K, Milczewska K (2009) Inverse gas chromatography as a source of physiochemical data. J Chromatogr A 1216:1551-1566

Walton K, Snurr R (2007) Applicability of the BET method for determining surface areas of microporous metal-organic frameworks. J Am Chem Soc 129:8552-8556

Wang H, Zhu E, Yang J, Zhou P, Sun D, Tang W (2012) Bacterial cellulose nanofiber-supported polyaniline nanocomposites with flake-shaped morphology as supercacitor electrodes. J Phys Chem 116:13013-13019 